\title{
Epidemiologia da morte materna e o desafio da qualificação da assistência
}

Epidemiology of maternal death and the challenge of care training Epidemiología de la muerte materna y el desafío de la cualificación de la atención

\author{
Janaina Aparecida Tintori' ic https://orcid.org/0000-0002-0466-3720 \\ Lise Maria Carvalho Mendes ${ }^{1}$ (D) https://orcid.org/0000-0001-9325-8382 \\ Juliana Cristina dos Santos Monteiro' ${ }^{1}$ il htps://orcid.org/0000-0001-6470-673X \\ Flávia Gomes-Sponholz ${ }^{1}$ il https://orcid.org/0000-0003-1540-0659
}

Como citar:

F. Epidemiologia da morte materna e o desafio

da qualificação da assistência. Acta Paul Enferm. 2022;35:eAPE00251.

DOI

http://dx.doi.org/10.37689/acta-ape/2022A000251

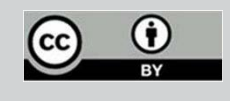

Descritores

Mortalidade materna; Qualidade da assistência à saúde; Saúde da mulher; Epidemiologia

Keywords

Maternal mortality; Quality of Health Care; Women's Health; Epidemiology

Descriptores

Mortalidad materna; Calidad de la atención de salud; Salud de la mujer; Epidemiología

Submetido 12 de Fevereiro de 2020

Aceito

23 de Março de 2021

Autor correspondente

Flávia Gomes-Sponholz

E-mail: flagomes@usp.br Orcid 0000-0003-1540-0659

Editor Associado (Avaliação pelos pares): Rosely Erlach Goldman (https://orcid.org/0000-0003-4011-1875) Escola Paulista de Enfermagem, Universidade Federal de São Paulo, São Paulo, SP, Brasil

\section{Resumo}

Objetivo: Descrever os óbitos maternos declarados e identificar o perfil epidemiológico das mulheres que foram a óbito em seu ciclo gravídico-puerperal e analisar as variáveis relacionadas à assistência no pré-natal e parto.

Métodos: Esta é uma pesquisa com delineamento retrospectivo com abordagem quantitativa do tipo levantamento. A população estudada foi constituída por mulheres que foram a óbito em seu período gravídicopuerperal, residentes em um dos 26 municípios da área de abrangência do Departamento Regional de Saúde de Ribeirão Preto, no período de 2011 a 2016. Foram analisados dados secundários obtidos via Departamento de Informática do Sistema Único de Saúde. Foram analisadas variáveis sociodemográficas, relativas ao óbito, ao pré-natal e parto e à rede de atenção à saúde. Os dados foram analisados de modo descritivo com a análise univariada.

Resultados: Foram encontrados registros de 36 óbitos maternos no período de 2011 a 2016, a maioria dos óbitos ocorreu em mulheres na faixa etária de 20 a 29 anos $(63,9 \%)$, com média de idade de 28,1 anos, sendo a maioria solteira $(50 \%)$, cor branca $(66,7 \%)$, primípara $(41,7 \%)$, com renda $(30 \%)$. 0 acesso ao prénatal foi perceptível na captação precoce $(72,2 \%)$ e no número de consultas durante o pré-natal. A principal via de parto foi a cesárea (52,8\%). As mortes maternas obstétricas diretas resultaram em $77,8 \%$ dos óbitos, sendo as principais causas: hipertensão, infecção e hemorragia.

Conclusão: 0 presente estudo mostrou que a maioria dos óbitos maternos ocorreu em mulheres na faixa etária de 20 a 29 anos, solteiras, de cor branca e primigestas. Foram perceptíveis a captação precoce e 0 adequado número de consultas durante o pré-natal. A classificação da maioria das mortes foi obstétrica direta, sendo hipertensão, infecção e hemorragia as principais causas. Foi possível conhecer a estrutura de redes e verificar uma boa cobertura de atenção primária e de atenção hospitalar para assistência ao parto.

\section{Abstract}

Objectives: To describe reported maternal deaths, identify the epidemiological profile of women who died during their pregnancy-postpartum cycle, and analyze the variables related to prenatal and childbirth care.

Methods: Retrospective quantitative survey. The studied population was women who died during their pregnancy-postpartum cycle and lived in one of the 26 municipalities in the area covered by the Ribeirão Preto Regional Health Department from 2011 to 2016. Secondary data obtained via the Department of Informatics of the Brazilian Unified Health System, including sociodemographic information and variables related to death, prenatal and childbirth, and the healthcare network, were treated by using descriptive statistics and univariate analysis. 
Results: Records of 36 maternal deaths were found for the period between 2011 and 2016. Most deaths occurred in women from 20 to 29 years old (63.9\%). The average age of the examined women was 28.1 years, and most were single (50\%), white (66.7\%), primiparous (41.7\%), and had an income source (30\%). Access to prenatal care was perceptible because of the existence of early recruitment $(72.2 \%)$ and the number of prenatal appointments. The main mode of delivery was cesarean (52.8\%). Direct obstetric causes of maternal death resulted in $77.8 \%$ of the occurrences, and the main causes were hypertension, infection, and bleeding.

Conclusion: The present study showed that most maternal deaths occurred in single, white, and primiparous women from 20 to 29 years old. Early recruitment and adequate number of prenatal appointments stood out. The classification of most deaths was direct obstetric, and hypertension, infection, and bleeding were the main causes. The present study exposed the network structure present in the healthcare sphere at issue and showed satisfactory primary healthcare and hospital coverage in childbirth care.

\section{Resumen}

Objetivo: Describir las defunciones maternas declaradas, identificar el perfil epidemiológico de las mujeres que fallecieron durante el embarazo 0 el puerperio y analizar las variables relacionadas con la atención prenatal y el parto.

Métodos: Se trata de un estudio con diseño retrospectivo y enfoque cuantitativo tipo recopilación. La población estudiada estuvo compuesta por mujeres que fallecieron durante el embarazo o el puerperio, residentes en algunos de los 26 municipios del área de cobertura del Departamento Regional de Salud de Ribeirão Preto, en el período de 2011 a 2016. Se analizaron datos secundarios obtenidos mediante el Departamento de Informática del Sistema Único de Salud. Se analizaron variables sociodemográficas, relativas a la defunción, a la atención prenatal y parto y a la red de atención en salud. Los datos se analizaron de modo descriptivo con el análisis univariado.

Resultados: Se encontraron registros de 36 defunciones maternas en el período de 2011 a 2016, la mayoría de las defunciones ocurrió en mujeres dentro del grupo de edad de 20 a 29 años (63,9 \%), con promedio de edad de 28,1 años, la mayoría soltera (50 \%), blanca (66,7 \%), primípara (41,7 \%), con ingresos (30\%). El acceso a la atención prenatal fue detectado mediante la captación temprana $(72,2 \%$ y y el número de consultas prenatales. La principal vía de parto fue la cesárea (52,8\%). Las muertes maternas obstétricas directas representaron el 77,8 \% de las defunciones, y las principales causas fueron: hipertensión, infección y hemorragia.

Conclusión: El presente estudio mostró que la mayoría de las defunciones maternas ocurrió en mujeres dentro del grupo de edad de 20 a 29 años, solteras, blancas y primíparas. Se detectó la captación temprana y un número adecuado de consultas prenatales. La clasificación de la mayoría de las muertes fue obstétricas directa, y las principales causas fueron hipertensión, infección y hemorragia. Fue posible conocer la estructura de redes y verificar una buena cobertura de atención primaria y de atención hospitalaria para asistencia al parto.

\section{Introdução}

A morte materna é definida pela Classificação Internacional de Doenças (CID10) como o óbito de uma mulher na gestação, no parto ou 42 dias após o parto, devido a qualquer causa relacionada ou agravada pelo período, excluindo causas acidentais ou incidentais. ${ }^{(1)}$

Segundo o Grupo Interinstitucional de Avaliação de Mortalidade Materna das Nações Unidas, em 2015 havia uma estimativa de 303.000 mortes maternas no mundo, perfazendo uma razão de mortalidade materna (RMM) de 216/100.000 nascidos vivos (NV), a maioria evitável e ocorreu em países em desenvolvimento. ${ }^{(2)}$ A maioria das mortes maternas seria evitável se as mulheres tivessem tratamento e cuidados adequados, incluindo atendimento pré-natal por profissionais de saúde bem capacitados, assistência durante o parto e apoio nas primeiras semanas após o parto. ${ }^{(3)}$

A mortalidade materna é um potente indicador de saúde que reflete as condiçóes sociais, econômicas e de qualidade de vida das pessoas que vivem em um determinado local. ${ }^{(4)}$ Reduzir a ocorrência de mortes maternas tem sido uma prioridade mundial e está incluída nas metas dos Objetivos de Desenvolvimento Sustentável e na Agenda 2030 da Organização das Nações Unidas. Investimentos e atenção contínuos são necessários para atingir a meta global de menos de 70 mortes maternas por 100.000 NV até 2030, o que poderia salvar mais de um milhão de vidas ao longo de uma década. ${ }^{(3)}$ Atingir essa meta é muito importante para melhores indicadores de saúde, mas muito mais por reduzir barreiras de acesso das mulheres a serviços de saúde e reduzir iniquidades. Uma condição imprescindível para avanços na redução de mortes maternas é entender as causas das mortes para decisóes efetivas sobre políticas e programas de saúde.

O Departamento Regional de Saúde de Ribeirão Preto (DRS XIII) é um dos 17 Departamentos Regionais de Saúde do Estado de São Paulo e conta com uma população estimada de 1.327.989 habitantes. ${ }^{(5)}$ Composto por 26 municípios, o DRS XIII é dividido em três regióes de saúde: Aquífero Guarani (10 municípios), Horizonte Verde (nove 
municípios) e Vale das Cachoeiras (sete municípios). O DRS XIII tem 20 maternidades, sendo 13 públicas e sete privadas. A Rede Cegonha foi implementada no DRS XIII, conforme portaria ministerial, para reorganizar a assistência em saúde para mulheres e crianças; propóe o monitoramento de alguns indicadores de saúde e visa o acesso a uma rede de cuidados, habilitação e custeio de leito.

A RMM no período de 2010 a 2016 no DRS XIII apresenta uma média de 30,59/100.000 NV, sendo que os anos de 2015 e 2016 chamaram a atenção por estarem acima dessa média, ficando em 63,37 e 51,95 por $100.000 \mathrm{NV}$, respectivamente. (6) Justificamos a realização do presente estudo, a partir da constatação do aumento do número dessas mortes na regiáo do DRS XIII. Entendemos ser importante descrever as causas de óbitos maternos e avaliar a assistência prestada, destacando a necessidade de intervenção numa população onde a morte materna é em sua maioria evitável, evidenciando um grande impacto familiar e social.

Os objetivos do estudo foram descrever os óbitos maternos declarados ocorridos nos municípios da área de abrangência do DRS XIII; identificar o perfil epidemiológico das mulheres que foram a óbito em seu ciclo gravídico-puerperal e analisar as variáveis relacionadas à assistência no pré-natal e parto.

\section{Métodos}

Esta é uma pesquisa com delineamento retrospectivo com abordagem quantitativa do tipo levantamento.

A população estudada foi constituída por mulheres que foram a óbito em seu período gravídico-puerperal, residentes em um dos 26 municípios da área de abrangência do DRS XIII, no período de 2011 a 2016.

Foram analisados dados secundários obtidos via Departamento de Informática do Sistema Único de Saúde (DATASUS) por meio dos sistemas: Sistema de Informação de Nascidos Vivos (SINASC) e Sistema de Informação de Mortalidade (SIM). Os dados de estrutura de redes foram obtidos pelo Cadastro Nacional de Estabelecimentos de Saúde
(CNES), sendo esses dados de estabelecimentos do Sistema Único de Saúde (SUS) e não SUS. ${ }^{(7)}$

Foram analisadas as seguintes variáveis: sociodemográficas (faixa etária, situação conjugal, cor, escolaridade, ocupação e procedência); relativas ao óbito (local do óbito, momento do ciclo gravídico-puerperal em que a morte ocorreu, assistência médica durante a doença que ocasionou o óbito, diagnóstico confirmado por necropsia e causa da morte materna obstétrica); relativas ao pré-natal e parto (local do pré-natal, número de gestaçôes, captação precoce, número de consultas de pré-natal, via de parto, profissional que assistiu ao parto); relativas à rede de atenção à saúde (identificação do percentual de cobertura de atenção básica e saúde suplementar por regiôes de saúde do DRS XIII, identificação das maternidades, números de leitos obstétricos de risco habitual e de gestação de alto risco, e total de leitos de unidade de terapia intensiva adulto).

Após autorização pelo Centro de Informações Estratégicas em Saúde (CIEVS) da Coordenadoria de Controle de Doenças (CCD) da Secretaria de Estado de Saúde de São Paulo (SES-SP), os dados foram coletados das Declaraçóes Óbito e ficha síntese de investigação que estão sob domínio do Sistema de Informação de Mortalidade.

Os dados foram analisados de modo descritivo com a análise univariada. As variáveis qualitativas foram apresentadas na forma de distribuição de frequências absolutas (n) e relativas (\%); e para as variáveis quantitativas foram calculados valores de média e mediana, desvios padrão e valores máximos e mínimos.

$\mathrm{O}$ projeto de pesquisa foi aprovado pelo Comitê de Ética em Pesquisa sob o número CAAE 79532617.0.0000.5393 e encaminhado ao CIEVS/ CCD da Secretaria de Estado de Saúde de São Paulo, a fim de atender às recomendaçóes contidas na resolução no 466/12 do Conselho Nacional de Saúde e das Diretrizes e Normas Regulamentadoras de Pesquisa envolvendo Seres Humanos.

\section{Resultados}

Foram encontrados no período de 2011 a 2016, o registro de 36 óbitos maternos de residentes nos 
municípios da área de abrangência do DRS XIII. A distribuição da razão de mortalidade materna do DRS XIII nos últimos seis anos foi $27,44 / 100.000$ NV em 2011, 16,54/100.000 NV em 2012, $16,74 / 100.000 \mathrm{NV}$ em 2013, 21,43/100.000 NV em 2014, 63,37/100.000 NV em 2015 e 51,95/100.000 NV em 2016.

\section{Mortalidade materna segundo perfil epidemiológico}

Observando o perfil de morte dessas mulheres, 23 óbitos $(63,9 \%)$ ocorreram em mulheres na faixa etária de 20 a 29 anos, seguido de 11 óbitos $(30,6 \%)$ na faixa etária de 30 a 39 anos, e dois óbitos $(5,6 \%)$ nas mulheres abaixo de 20 anos. Nenhum óbito foi encontrado em mulheres acima de 40 anos, sendo a idade mínima de 18 anos e a idade máxima de 39 anos, a média de idade dessas mulheres foi de 28,1 anos. Analisando a situação conjugal, 18 mulheres (50\%) eram solteiras e $12(33,4 \%)$ casadas ou tinham união estável, quanto à cor, 24 mulheres (66,7\%) eram brancas, seguidas de cinco $(13,9 \%)$ pardas e cinco $(13,9 \%)$ pretas. O perfil de escolaridade mostrou que 19 mulheres $(52,8 \%)$ tinham oito anos ou mais de estudo e cinco $(13,9 \%)$ tinham ensino superior completo. Em relação a ocupação, o registro dessa informação demonstrou que 11 mulheres (30\%) possuíam renda, considerando que possuíam um código de Classificação Brasileira de Ocupação preenchida para esse campo na DO, já em 20 (55,6\%) registros essa informação foi preenchida como ignorada e cinco $(13,9 \%)$ registros estavam em branco, ou seja, sem preenchimento do dado.

\section{Mortalidade materna segundo assistência no pré-natal e parto}

Quanto ao local de realização do pré-natal, 13 mulheres $(36,1 \%)$ acompanharam suas gestaçóes na atenção primária, seguidas de nove mulheres $(19,4 \%)$ que estavam inseridas no acompanhamento de alto risco; e cinco $(13,9 \%)$ que realizaram o pré-natal na saúde suplementar. Das 36 mulheres, 15 estavam na sua primeira gestação $(41,7 \%), 10$ $(27,8 \%)$ eram secundigestas e $11(30,6 \%)$ eram multigestas. Ao verificar o início do pré-natal em relação à idade gestacional, 26 mulheres $(72,2 \%)$ iniciaram o acompanhamento antes da $12^{\mathrm{a}}$ semana, e em relação ao número de consultas, 18 mulheres $(50 \%)$ realizaram sete ou mais consultas, seguidas de sete gestantes $(19,4 \%)$ com quatro a seis consultas e cinco $(13,9 \%)$ com uma a três consultas. A principal forma de resoluçáo da gravidez foi a cesárea, sendo 19 mulheres (52,8\%) submetidas ao procedimento cirúrgico.

Segundo dados analisados, 33 mulheres $(91,7 \%)$ morreram em ambiente hospitalar, 28 (77,8\%) tiveram assistência médica declarada no momento do óbito e 20 mulheres $(55,6 \%)$ foram encaminhadas para necropsia (Tabela 1).

Tabela 1. Caracterização dos óbitos por local de ocorrência e condição de assistência

\begin{tabular}{lc}
\hline Variável & Frequência(\%) \\
\hline Local do óbito & \\
Hospital & $33(91,7)$ \\
Outro estabelecimento de saúde & $1(2,8)$ \\
Domicílio & $2(5,6)$ \\
Recebeu assistência médica & \\
Sim & $28(77,8)$ \\
Não & $3(8,3)$ \\
Ignorado & $5(13,9)$ \\
Encaminhado para necropsia & \\
Sim & $20(55,6)$ \\
Não & $15(41,7)$ \\
Ignorado & $1(2,8)$ \\
\hline
\end{tabular}

Fonte: SES SP/ CCD/ CIEVS, 2018.

\section{Mortalidade materna segundo classificação, causa obstétrica e momento do óbito}

Para a análise das causas das mortes foi utilizada a lista de três caracteres da $10^{a}$ Revisão da Classificação de Doenças e Problemas Relacionados à Saúde CID - 10, capítulo XV, formando agrupamentos e códigos. Para o agrupamento morte materna direta, foram considerados os códigos Hipertensão O11, O13, O14, O15 e O16; Hemorragias O45 e O72; Infecção O23, O85 e O86 e demais causas O43, O73, O75 e O90. Para as causas obstétricas indiretas, foram considerados os códigos: Doença para HIV B20; Complicaçóes no puerpério O98 e O99 e Hipertensão preexistente $\mathrm{O} 10$, sendo que 28 mulheres $(77,7 \%)$ morreram por causas obstétricas diretas. $\mathrm{Na}$ distribuição desses óbitos no ciclo gravídico-puerperal, observou-se que o período de maior risco foi o pós-parto com 25 mortes $(69,5 \%)$, sendo oito $(22,2 \%)$ durante a gestação, dois $(5,5 \%)$ 
Tabela 2. Distribuição dos óbitos maternos segundo classificação, causa obstétrica e momento do óbito

\begin{tabular}{|c|c|c|c|c|c|}
\hline \multirow{2}{*}{ Causa básica do óbito - CID 10} & \multirow[b]{2}{*}{ Gestação } & \multicolumn{3}{|c|}{ Momento do óbito } & \multirow[b]{2}{*}{$\mathrm{n}(\%)$} \\
\hline & & Parto & Puerpério imediato & Puerpério tardio & \\
\hline Morte materna obstétrica indireta & & & & & $7(19,5)$ \\
\hline Hipertensão preexistente (010) & & & 1 & & $1(2,8)$ \\
\hline Complicações no puerpério (098-099) & 2 & & 3 & & $5(13,9)$ \\
\hline Doença por HIV (B20) - aborto & 1 & & & & $1(2,8)$ \\
\hline Morte materna obstétrica direta & & & & & $28(77,7)$ \\
\hline Transtornos hipertensivos na gravidez, parto e puerpério (011-016) & 4 & & 3 & & $7(19,4)$ \\
\hline Infecção na gravidez e puerpério (023-085-086) & 1 & & 6 & & $7(19,4)$ \\
\hline Hemorragia (045-072) & & 2 & 5 & & $7(19,4)$ \\
\hline Demais causas (043-073-075-090) & 1 & & 6 & & $7(19,4)$ \\
\hline Morte materna obstétrica não especificada & & & & & $1(2,8)$ \\
\hline Morte obstétrica de causa $\mathrm{NE}^{\star}(095)$ & & & & 1 & $1(2,8)$ \\
\hline Total & 9 & 2 & 24 & 1 & $36(100)$ \\
\hline
\end{tabular}

no parto, $24(66,7 \%)$ no puerpério imediato (até 10 dias após o parto) e um $(2,8 \%)$ no puerpério tardio (após 10 dias do parto) e uma (2,8\%) morte por aborto (Tabela 2).

\section{Discussão}

No presente estudo foram registradas e declaradas 36 mortes de mulheres no ciclo gravídico-puerperal, no período de 2011 a 2016. Esses números chamam a atenção pelo aumento da ocorrência dos óbitos a partir de 2015 e mantendo-se em 2016, demonstrando uma linha de ascendência. Fazendo uma análise da série histórica estudada, a RMM do DRS XIII no período de 2011 a 2014 apresentou índices abaixo da RMM do estado de São Paulo. Porém, em 2015 os números de óbitos maternos no DRS XIII triplicaram, chegando a uma RMM de 63,37/100.000 NV, superando o estado de São Paulo em 25\%, que no mesmo ano teve RMM de 47,53/100.000 NV. ${ }^{(8)}$ Preocupa-nos essa informação, pois nos anos seguintes ao estudado, 2017 e 2018, esse platô se manteve no DRS XIII com RMM de 55,56/100.000 NV e 60,69/100.000 $\mathrm{NV}$ respectivamente, sem perspectiva de queda. ${ }^{(6)}$ A OMS alerta para os parâmetros das altas taxas de mortalidade materna (RMM acima de 50/100.000 $\mathrm{NV}$ ), e os resultados encontrados no DRS XIII, coloca a regiáo com classificação alta e preocupante.

Neste estudo, a maioria dos óbitos maternos ocorreu em mulheres jovens, na faixa etária de 20 a 29 anos, sendo a maioria solteira, da cor branca e com oito ou mais anos de estudo. A caracterização dos óbitos corrobora outros estudos considerando a faixa etária, situaçáo conjugal, ocupação e escolaridade. ${ }^{(9-11)}$ Porém, segundo outras evidências, a mortalidade materna é mais frequente entre mulheres negras, condição explicada pela associação do difícil controle pressórico das doenças hipertensivas nessa população, dificuldade de acesso aos serviços de saúde e pela baixa qualidade da assistência. ${ }^{(10,12)}$ Um estudo retrospectivo americano, concluiu que mulheres afro-americanas têm uma razão de mortalidade materna quatro vezes maior que as mulheres americanas brancas. ${ }^{(13)}$ Quanto a situação conjugal, pesquisadores concordam que a presença do companheiro tem sido interpretada como possibilidade de maior suporte emocional durante a gravidez e parto. $^{(14)}$

Outro aspecto identificado no presente estudo foi o acesso garantido das gestantes ao pré-natal, seja através da atenção primária ou pela saúde suplementar, a maioria das mulheres teve acesso ao acompanhamento pré-natal iniciando ainda no primeiro trimestre, perfazendo seis ou mais consultas, conforme preconizado pelas diretrizes da Rede Cegonha, porém com desfecho desfavorável. Tal fato chama atenção de que mesmo garantindo os indicadores do Ministério da Saúde, como captação precoce, número de consultas e acesso, que são indicadores quantitativos, a qualidade náo é mensurada por esses dados na atenção obstétrica.

Segundo o estudo Nascer no Brasil, 98,7\% das gestantes tiveram cobertura elevada da assistência pré-natal, com $75,8 \%$ das mulheres iniciado o pré-natal precocemente e $73,1 \%$ compareceram a seis ou mais consultas. ${ }^{(15)} \mathrm{A}$ pesquisa enfatizou ainda 
que a assistência ao pré-natal deve ser acolhedora de modo a proporcionar confiança e vinculo da gestante com a unidade. ${ }^{(15)}$

Quanto ao desfecho, quase a totalidade das mulheres deste estudo morreu em uma instituição hospitalar, teve assistência médica no momento do óbito e foi encaminhada para necropsia. O encaminhamento dessas mulheres para necropsia é de suma importância, contribuindo assim para identificação das possíveis causas e subsidiando adoção de medidas que possam evitar a sua reincidência.

Entretanto, o método utilizado neste estudo não nos permitiu acesso aos resultados da necropsia para a confirmação da causa básica do óbito. Porém, todas as mortes maternas ocorridas nos municípios da área de abrangência do DRS XIII são apresentadas e discutidas no Comitê Regional de Vigilância a Morte Materna, Infantil e Fetal. Este, assim como as demais Comissões ou Comitês de Prevenção e Combate à Morte Materna, tem caráter educativo e é responsável por identificar a magnitude da mortalidade materna, suas causas e fatores que a determinaram, avaliando os resultados da assistência prestada às gestantes e puérperas. Após a análise das investigaçôes dos óbitos, o Comitê encaminha recomendaçôes, se necessário, aos gestores de todos os níveis de atenção a saúde para ciência e devidas providências.

O período de maior risco foi o puerpério, seguido da gestação. Corrobora o estudo realizado na Índia, que alerta para a alta mortalidade no primeiro e segundo dia após o nascimento. ${ }^{(16)}$ Nesse contexto, é essencial a qualificação da equipe para reconhecimento das emergências e intervenção imediata às complicaçóes. ${ }^{(17,18)}$

Analisando os tipos de parto, o presente estudo observou que mais da metade das mulheres foi submetida à cesariana. Uma meta-análise recente concluiu que mulheres que foram submetidas à cesariana apresentaram 3,1 vezes mais chances de morrer do que as que tiveram parto normal. ${ }^{(18)} \mathrm{A}$ cesariana pode contribuir para o aumento dos óbitos maternos e para a morbidade materna grave, devido a exposição da mulher a riscos inerentes de uma cirurgia, como hemorragias, infecçôes, reações anestésicas, embolias, entre outros, somente devendo ser indicado em situações de comprovada morbidade obstétrica e riscos fetais. ${ }^{(19)}$

Em relação à paridade, as mortes ocorreram mais entre as primigestas. A maioria das mortes em primigestas não corresponde aos achados na literatura, que apontam a multiparidade como fator de risco, principalmente devido maiores chances de hemorragias, considerando que o aumento das mortes em primigestas podem estar associadas à comorbidades. ${ }^{(20,21)}$

Quanto às causas das mortes maternas, a maioria dos óbitos foi por causa obstétrica direta, sendo distribuídos em complicações da hipertensão, hemorragia, infecção e outras causas que complicam a gravidez, o parto e ou o puerpério. Um estudo realizado no estado de São Paulo mostrou a transição da principal causa de morte materna no estado entre os anos de 2010 e 2015, diminuindo os óbitos por distúrbios hipertensivos e aumentando a morte das mulheres por hemorragias. Essas causas chamaram a atenção, pois poderiam ser evitadas por meio de integração das redes de atenção à saúde e ambientes hospitalares prioritariamente organizados, prontos e qualificados. ${ }^{(11)}$ As mortes causadas por distúrbios hipertensivos também merecem análise, principalmente quando se instala nas suas formas mais graves, como a eclâmpsia e a síndrome HELLP.

Em relação à morte obstétrica indireta o presente estudo encontrou como causas: outras doenças classificadas em outra parte (que pode ser por anemia, sistema circulatório, imunológico, respiratório), seguidas de hipertensão crônica, doenças infecciosas e parasitárias e doença pelo vírus da imunodeficiência humana. Na literatura, o número óbitos por causa indireta encontra-se mais elevado em hospitais de referência para gestação de alto risco. ${ }^{(22)}$

Mortes obstétricas indiretas poderiam ser evitadas com planejamento familiar e reprodutivo de qualidade. Os profissionais de saúde têm um papel importante no reconhecimento precoce de sinais e sintomas de complicações durante todo o ciclo grávido-puerperal. A utilização do histórico obstétrico da mulher pode ser uma ferramenta de risco preditivo na evitabilidade da morte materna. ${ }^{(13)}$ Outros autores reforçam esse alerta, destacando patologias pregressas importantes para atenção na condução 
da gestação atual e parto, como a eclâmpsia, diabetes, obesidade e gestação múltipla, devido essas causas apresentarem maior associação com a morte materna indireta. ${ }^{(23)}$

Vários estudos demonstraram que a mortalidade materna está diretamente relacionada à qualidade dos cuidados médicos e obstétricos ofertados durante a gestação, parto e puerpério. Independentemente de outros fatores, é imprescindível a garantia de atenção obstétrica humanizada, especializada, com equipe qualificada e preparada para identificação e manejo de situaçóes de emergências. Todas essas ações devem estar alinhadas a uma gestão focada na redução da morbimortalidade materna. ${ }^{(24,25)}$

No contexto das redes de atenção como arranjos organizativos de ações e serviços de saúde e tendo a regionalização como uma diretriz do SUS, focado na saúde da mulher, o cuidado à usuária deve ser integral, universal, resolutivo e descentralizado. ${ }^{(26)}$ Desta forma, o DRS XIII, baseado nas recomendaçóes da Rede Cegonha, criou a Rede Materna Regional, pactuada em instâncias colegiadas, reunindo gestores e técnicos de saúde, através de Câmaras Técnicas Regionais e Grupos Técnicos de Trabalho para avaliação e construção da mesma. A criação de colegiados, espaços coletivos de gestão e de mobilização tem sido uma das principais ofertas da Rede Cegonha para discussóes técnicas e políticas pautadas na saúde das mulheres.

Conhecendo a estrutura de redes da região, o DRS XIII apresenta boa cobertura de atenção básica nas três regióes de saúde. Estudos referenciam a atenção primária como garantia de implementação de estratégias de promoção da saúde reprodutiva e sexual, atividades educativas relacionadas ao pré-natal, puerpério e planejamento familiar. Qualquer falha de assistência nessa estrutura pode ocasionar o aparecimento de complicaçóes, contribuindo consequentemente para o surgimento da morbimortalidade materna. ${ }^{(25)}$

O DRS XIII conta ainda com uma rede hospitalar de 20 maternidades para atenção à gestação, e possui em seu território duas maternidades públicas para atendimento de alta complexidade. No Paraná, ao investigar a rede hospitalar de assistência ao parto com amplo atendimento regional e cobertura esta- dual, foi verificada elevada taxa de óbitos maternos evitáveis, o que permitiu inferir que prováveis falhas advinham de deficiência de infraestrutura adequada, capacitação dos profissionais, e deficiência de adoção de práticas baseadas em evidências científicas preconizadas por organismos internacionais e nacionais. ${ }^{(9)}$

No interior de Minas Gerais, pesquisadores avaliaram as expectativas e percepçôes de mulheres em relação ao parto, e o estudo evidenciou que as gestantes esperavam atendimento de qualidade, com profissionais humanos e acolhedores. ${ }^{(27)}$ Tais expectativas e percepçóes vão de encontro a esta discussão, considerando que as mulheres também compreendem que quanto mais o serviço é especializado e qualificado menos chances de desfechos desfavoráveis.

Os resultados apresentados resumem que a morte materna continua sendo um desafio para a assistência obstétrica na região. É fundamental que os profissionais e instituições estejam adequados, comprometidos e alicerçados em evidências científicas, fazendo-se urgente e necessária a mudança de modelo assistencial prestado a essas mulheres e suas famílias.

\section{Conclusão}

O presente estudo mostrou que a maioria dos óbitos maternos ocorreu em mulheres na faixa etária de 20 a 29 anos, solteiras, de cor branca e primigestas. Foram perceptíveis a captação precoce e o adequado número de consultas durante o pré-natal. A classificação da maioria das mortes foi obstétrica direta, sendo hipertensão, infecção e hemorragia as principais causas. Foi possível conhecer a estrutura de redes e verificar uma boa cobertura de atenção primária e de atenção hospitalar para assistência ao parto na área de abrangência do DRS XIII.

\section{Colaborações}

Tintori JA, Mendes LM, Monteiro JC e GomesSponholz F contribuíram com a concepção do es- 
tudo, análise e interpretação dos dados, redação do artigo, revisão crítica relevante do conteúdo intelectual e aprovação da versão final a ser publicada.

\section{Referências}

1. World Health Organization (WHO). Trends in maternal mortality: 2000 to 2017: estimates by WHO, UNICEF, UNFPA, World Bank Group and the United Nations Population Division. Geneva: WHO; 2019.

2. Ozimek JA, Kilpatrick SJ. Maternal mortality in the twenty-first century. Obstet Gynecol Clin North Am. 2018;45(2):175-86. Review.

3. United Nations. The sustainable development goals report 2019 [Internet]. New York: United Nations; 2019 [cited 2019 Apr 5]. Available from: https:// unstats.un.org/sdgs/report/2019/The-Sustainable-Development-GoalsReport-2019.pdf

4. Chou VB, Walker N, Kanyangarara M. Estimating the global impact of poor quality of care on maternal and neonatal outcomes in 81 low- and middle-income countries: a modeling study. PLOS Med. 2019;16(12):e1002990.

5. Brasil. Ministério da Saúde. Departamento de informática do Sistema Único de Saúde (DATASUS). Informações de Saúde (TABNET). Censo demográfico 2010. População residente, por sexo - Censo. População total DRS XIII [Internet]. Brasília (DF): Ministério da Saúde; 2010 [citado 2018 Ago 1]. Disponível em: http://tabnet.saude.sp.gov.br/tabcgi. exe?tabnet/ind3_censo.def

6. Brasil. Ministério da Saúde. Departamento de informática do Sistema Único de Saúde (DATASUS). Informações de Saúde (TABNET). Razão de mortalidade materna. Matriz de indicadores de saúde: razão de mortalidade materna [Internet]. Brasília (DF): Ministério da Saúde; 2010 [citado 2019 Abr 5]. Disponível em: http://tabnet.saude.sp.gov. br/tabcgi.exe?tabnet/ind14_matriz.def

7. Brasil. Ministério da Saúde. Departamento de informática do Sistema Único de Saúde (DATASUS). Cadastro Nacional de Estabelecimentos de Saúde [Internet]. Brasília (DF): Ministério da Saúde; 2021 [citado 2017 Jun 5]. Disponível em: http://datasus1.saude.gov.br/sistemas-eaplicativos/cadastros-nacionais/cnes

8. Brasil. Governo Federal. Ministério da Saúde. Portal Brasil: Saúde. Sistema Único de Saúde [Internet]. Brasília (DF): Ministério da Saúde; 2021 [citado 2018 Jul 30]. Disponível em: http://portalms.saude.gov. br/sistema-unico-de-saude

9. Martins AC, Silva LS. Epidemiological profile of maternal mortality. Rev Bras Enferm. 2018;71(Suppl 1):677-83.

10. Mendes JD. A mortalidade materna no estado de São Paulo, Brasil. BEPA Bol Epidemiol Paul. 2018;15(173):3-9.

11. Moraes MM, Quaresma MA, Oliveira US, Silveira MM. Gestational risk classification based on maternal death profile 2008-2013: an experience report from the municipality of Porto Seguro, Bahia, Brazil. Epidemiol Serv Saúde. 2019;28(3):e2018491.

12. Cox RS, Faan R. Global maternal mortality rate declines - except in America. Nurs Outlook. 2018;66(5):428-9.

13. Holdt Somer SJ, Sinkey RG, Bryant AS. Epidemiology of racial/ethnic disparities in severe maternal morbidity and mortality. Semin Perinatol. 2017;41(5):258-65. Review.

14. Ayala Quintanilla BP, Pollock WE, McDonald SJ, Taft AJ. Intimate partner violence and severe acute maternal morbidity in the intensive care unit: a case-control study in Peru. Birth. 2020;47(1):29-38.

15. Viellas EF, Domingues RM, Dias MA, Gama SG, Theme Filha MM, Costa JV, et al. Prental care in Brazil. Cad Saúde Pública. 2014;30(Suppl 1):S85-S100.

16. Kumari K, Srivastava RK, Srivastava M, Purwar N. Maternal mortality in rural Varanasi: delays, causes, and contributing factors. Indian J Community Med. 2019;44(1):26-30.

17. Lima MR, Coelho AS, Salge AK, Guimarães JV, Costa PS, Sousa TC, et al. Alterações maternas e desfecho gravídico-puerperal na ocorrência de óbito materno. Cad Saúde Colet. 2017;25(3):324-31.

18. Mascarello KC, Horta BL, Silveria MF. Maternal complications and cesarean section without indication: systematic review and metaanalysis Rev Saúde Pública. 2017;51:105.

19. Fahmy WM, Crispim CA, Cliffe S. Association between maternal death and cesarean section in Latin America: a systematic literature review. Midwifery. 2018;59:88-93. Review.

20. Maswime S, Buchmann E. A systematic review of maternal near miss and mortality due to postpartum hemorrhage. Int J Gynaecol Obstet. 2017;137(1):1-7. Review.

21. Fernandes BB, Nunes FB, Prudêncio PS, Mamede FV. Epidemiological research of the materna deaths and compliance with the fifth millennium development goal. Rev Gaúcha Enferm. 2015;36(Spe):192-9.

22. Soares VM, Souza KV, Azevedo EM, Possebon CR, Soares FF. Causas de mortalidade materna Segundo níveis de complexidade hospitalar. Rev Bras Ginecol Obstet. 2012;34(12):536-43.

23. Mersha AG, Abegaz TM, Seid MA. Maternal and perinatal outcomes of hypertensive disorders of pregnancy in Ethiopia: systematic review and meta-analysis. BMC Pregnancy Childbirth. 2019;19(1):458.

24. Main EK. Reducing maternal mortality and severe maternal morbidity through state-based quality improvement initiatives. Clin Obstet Gynecol. 2018;61(2):319-31.

25. Vieira LB, Gouveia HG, Wegner W, Gerhardt LM. The Millennium Development Goals and the social commitment of Nursing research [Editorial]. Rev Gaúcha Enferm. 2015;36(1):8-9.

26. Mendes EV. As redes de atenção à saúde. Brasília (DF): Organização Pan-Americana da Saúde; 2011. 549p.

27. Costa NS, Bracarense CF, Duarte JM, Paula MS, Simões A. Expectativas, percepções e opiniões de mulheres sobre 0 atendimento durante 0 parto. Rev Min Enferm. 2018;22:e-1129. 